\title{
An Analytical Study of Weakly Nonlinear Dynamics of a Walters' Liquid B around a Flexible Sheet Undergoing Super Linear Stretching
}

\author{
P. G. Siddheshwar, ${ }^{1}$ A. Chan, ${ }^{2}$ and U. S. Mahabaleswar ${ }^{3}$ \\ ${ }^{1}$ Department of Mathematics, Bangalore University, Central College Campus, Bangalore 560 001, India \\ ${ }^{2}$ Department of Civil Engineering, University of Nottingham, Malaysia Campus, Jalan Broga, \\ 43500 Semenyih, Malaysia \\ ${ }^{3}$ Department of Mathematics, Government First Grade College for Women, Hassan 573 201, India
}

Correspondence should be addressed to U. S. Mahabaleswar, ulavathi@gmail.com

Received 21 August 2011; Accepted 7 October 2011

Academic Editor: M. F. El-Sayed

Copyright (C) 2012 P. G. Siddheshwar et al. This is an open access article distributed under the Creative Commons Attribution License, which permits unrestricted use, distribution, and reproduction in any medium, provided the original work is properly cited.

The paper discusses the boundary layer flow of Walters' liquid B over a stretching sheet. The stretching is assumed to be a quadratic function of the coordinate along the direction of stretching. The study encompasses within its realm both Walters' liquid B and second order liquid. The velocity distribution is obtained by solving the nonlinear governing differential equation. Analytical expressions are obtained for stream function and velocity components as functions of the viscoelastic and stretching related parameters. It is shown that the viscoelasticity goes hand in hand with quadratic stretching in enhancing the lifting of the liquid as we go along the sheet.

\section{Introduction}

Polymer extrusion, drawing of copper wires, continuous stretching of plastic films, and artificial fibers, hot rolling, wire drawing, glass-fiber, metal extrusion, and metal spinning are some of the examples where the problem of a stretching sheet arises. Ever since the pioneering works of Sakiadis [1,2], several works have appeared to consider various aspects of the problem (see Siddheshwar and Mahabaleswar [3, 4], Andersson [5-7], Rollins and Vajravelu [8], Vleggar [9], Ming-I and Cha'o-Kuang [10], Kelly et al. [11], Vajravelu and Hadjinicolaou [12], Liao and Pop [13], Magyari et al. [14], Liao [15], and Dandapat and Gupta [16]). The core assumption in most of the reported problems is that the stretching is linearly proportional to the axial distance. This is valid provided the stretching process is delicate and slow, leading to the assumption of constant rate of stretching. It is not difficult to see that the above assumption is quite idealistic and impractical. In the strictest sense 


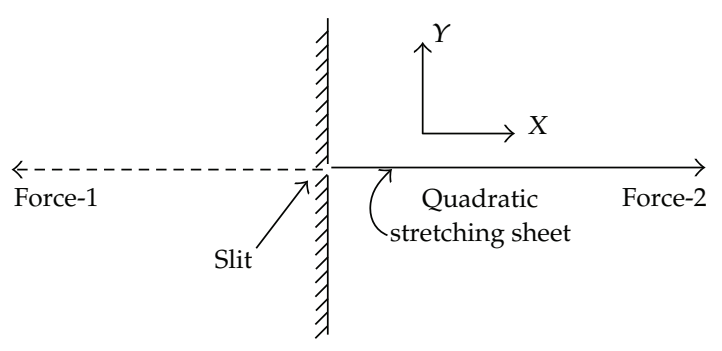

Figure 1: Schematic diagram of the stretching sheet problem.

the stretching has to be nonlinearly proportional to the axial distance. In the present paper, as a first step in the general modelling exercise, we make use of a simple quadratic stretching model.

\section{Mathematical Formulation}

We consider a steady state two-dimensional boundary layer flow of an incompressible isothermal viscoelastic liquid, of the type Walters' liquid B, over a quadratic stretching sheet (see Figure 1). The Walters' liquid B represents an approximation for short or rapidly fading memory liquids and is thus an approximation to first order in elasticity. The liquid is at rest and the motion is created by pulling the sheet on both ends with equal forces parallel to the sheet and with a speed $u$, which varies quadratically with the distance from the slit as $u=\alpha x+\beta x^{2}$. The resulting motion of the otherwise quiescent liquid is thus caused solely by the moving sheet. On assuming $\beta$, and thereby $\delta$, quite small we can make use of the boundary layer theory (see Rajagopal et al. [17]).

The steady two-dimensional conservation of mass and the momentum boundary layer equation for the quadratic stretching sheet problem involving Walters' liquid B are (see Beard and Walters [18]):

$$
\begin{gathered}
\frac{\partial u}{\partial x}+\frac{\partial v}{\partial y}=0 \\
u \frac{\partial u}{\partial x}+v \frac{\partial u}{\partial y}=v \frac{\partial^{2} u}{\partial y^{2}}-k_{0}\left\{u \frac{\partial^{3} u}{\partial x \partial y^{2}}+v \frac{\partial^{3} u}{\partial y^{3}}+\frac{\partial u}{\partial x} \frac{\partial^{2} u}{\partial y^{2}}-\frac{\partial u}{\partial y} \frac{\partial^{2} u}{\partial x \partial y}\right\}
\end{gathered}
$$

subject to the boundary conditions:

$$
\begin{gathered}
u=\alpha x+\beta x^{2} \quad \text { at } y=0, \\
v=\delta x \quad \text { at } y=0, \\
u=0 \quad \text { as } y \longrightarrow \infty .
\end{gathered}
$$

Here, $u$ and $v$ are the components of the liquid velocity in the $x$ and $y$ directions, respectively, $\mu$ is the limiting viscosity at small rates, and $k_{0}$ is the first moment of the distribution function 
of relaxation times. Further we assume $\beta$ is quite small, that facilitates the assumption of a weakly two-dimensional flow as considered in the paper.

As pointed out by Vleggaar [9], in a polymer processing application involving spinning of filaments without blowing, laminar boundary layer occurs over a relatively small length of the zone $0.0-0.5 \mathrm{~m}$ from the die which may be taken as the origin of Figure 1 . This is in fact the zone over which the major part of the stretching takes place. In such a process the initial velocity is low (about $0.3 \mathrm{~m} / \mathrm{s}$ ) but not very low, enough always to assume linear stretching. Thus a good approximation of the velocity of the sheet is $u=\alpha x+\beta x^{2}$ (at any rate for the first $10-60 \mathrm{~cm}$ of the spinning zone), where $\alpha$ and $\beta$ are the constants velocity gradients. We have adopted the quadratic stretching model in our problem. Using the dimensionless variables

$$
(X, Y)=\sqrt{\frac{\alpha}{v}}(x, y), \quad(U, V)=\frac{(u, v)}{\sqrt{\alpha \nu}}, \quad \beta^{*}=\frac{\beta}{\alpha} \sqrt{\frac{v}{\alpha}}, \quad \delta^{*}=\frac{\delta}{2 \alpha} .
$$

Equation (2.1) take the form

$$
\begin{gathered}
\frac{\partial U}{\partial X}+\frac{\partial V}{\partial Y}=0 \\
U \frac{\partial U}{\partial X}+V \frac{\partial U}{\partial Y}=\frac{\partial^{2} U}{\partial Y^{2}}-k_{1}\left\{U \frac{\partial^{3} U}{\partial X \partial Y^{2}}+V \frac{\partial^{3} U}{\partial Y^{3}}+\frac{\partial U}{\partial X} \frac{\partial^{2} U}{\partial Y^{2}}-\frac{\partial U}{\partial Y} \frac{\partial^{2} U}{\partial X \partial Y}\right\}
\end{gathered}
$$

where $k_{1}=\alpha k_{0} / \mu$ is the viscoelastic parameter. The parameter $k_{1}$ represents a measure of the relative importance of elastic and viscous effects and can thus be identified with the Weissenberg number.

Introducing the stream function $\psi(X, Y)$, we get

$$
U=\frac{\partial \psi}{\partial Y}, \quad V=-\frac{\partial \psi}{\partial X} .
$$

Using (2.5) in (2.6), we get

$$
\frac{\partial^{3} \psi}{\partial Y^{3}}+\frac{\partial(\psi, \partial \psi / \partial Y)}{\partial(X, Y)}+k_{1}\left\{\frac{\partial\left(\psi, \partial^{3} \psi / \partial Y^{3}\right)}{\partial(X, Y)}-\frac{\partial\left(\partial \psi / \partial Y, \partial^{2} \psi / \partial Y^{2}\right)}{\partial(X, Y)}\right\}=0 .
$$

The boundary conditions to be satisfied by $\psi$ can be obtained from (2.2), (2.3), and (2.5) as follows:

$$
\begin{gathered}
\frac{\partial \psi}{\partial Y}=X+\beta^{*} X^{2} \quad \text { at } Y=0, \\
-\frac{\partial \psi}{\partial X}=2 \delta^{*} X \quad \text { at } Y=0, \\
\frac{\partial \psi}{\partial Y}=0 \quad \text { as } Y \longrightarrow \infty .
\end{gathered}
$$


The similarity solution to (2.6), subject to (2.7), may be taken as

$$
\psi=X f(Y)-\delta^{*} X^{2} f^{\prime}(Y)
$$

where prime denotes differentiation with respect to $Y$. Substituting (2.8) into (2.6) and equating the coefficients of $X, X^{2}$, and $X^{3}$, we get the following three ordinary differential equations:

$$
\begin{gathered}
\left(f^{\prime}\right)^{2}-f f^{\prime \prime}=f^{\prime \prime \prime}-k_{1}\left\{2 f^{\prime} f^{\prime \prime \prime}-f f^{\prime \prime \prime \prime}-\left(f^{\prime \prime}\right)^{2}\right\}, \\
f^{\prime} f^{\prime \prime}-f f^{\prime \prime \prime}=f^{\prime \prime \prime \prime}-k_{1}\left\{f^{\prime} f^{\prime \prime \prime \prime}-f f^{\prime \prime \prime \prime \prime}\right\} \\
\left(f^{\prime \prime}\right)^{2}-f^{\prime} f^{\prime \prime \prime}=k_{1}\left\{f^{\prime} f^{\prime \prime \prime \prime \prime}-2 f^{\prime \prime} f^{\prime \prime \prime \prime}+\left(f^{\prime \prime \prime}\right)^{2}\right\} .
\end{gathered}
$$

Equation (2.10) turns out redundant as it can be obtained by differentiating (2.9) once with respect to $Y$. In the subsequent analysis we show that (2.9) can, in fact, be obtained from (2.11), by a suitable transformation, which in turn implies consistency. The boundary conditions, for solving (2.9) for $f$, given by (2.7) can be obtained in the form

$$
\begin{aligned}
& f^{\prime}(0)=1, \quad f^{\prime \prime}(0)=-s, \\
& f(0)=0, \\
& f^{\prime}(\infty)=0, \quad f^{\prime \prime}(\infty)=0,
\end{aligned}
$$

where $s=\beta^{*} / \delta^{*}$. One can easily see that (2.11) is a differential equation for $f^{\prime}(Y)$ and we can also verify that $f^{\prime}(Y)=e^{-s Y}$ is a solution of (2.11), and this satisfies the derivative boundary conditions in $(2.12 \mathrm{a})-(2.12 \mathrm{c})$. Thus an appropriate solution of $(2.9)$ is

$$
f(Y)=A+B e^{-s Y},
$$

which satisfies the boundary condition (2.12a)-(2.12c) provided

$$
A=\frac{\delta^{*}}{\beta^{*}}, \quad B=-\frac{\delta^{*}}{\beta^{*}}, \quad s=\frac{1}{A} .
$$

We also note that (2.13) can be a solution of the nonlinear differential equation (2.9) if and only if

$$
s=\frac{\beta^{*}}{\delta^{*}}=\frac{1}{\sqrt{1-k_{1}}} .
$$

We may now write $f(Y)$ from (2.13)-(2.15) as

$$
f(Y)=\sqrt{1-k_{1}}\left(1-e^{-Y / \sqrt{1-k_{1}}}\right) .
$$


Reverting to the symbol "s" we may easily see that

$$
f^{\prime}(Y)=1-s f(Y)
$$

Using this in (2.11) we can arrive at (2.9). This proves the "consistency" of the 3 equations (2.9)-(2.11) for $f(Y)$.

The expression for the streamline pattern of the flow in the region around the stretching sheet can be obtained from (2.8) as follows:

$$
\psi=X f(Y)-\delta^{*} X^{2} f^{\prime}(Y)=C
$$

where $C$ is a constant. The streamline $\psi=C$ can be written in the functional form as

$$
Y=\frac{1}{s} \operatorname{Ln}\left\{\frac{X / s+\delta^{*} X^{2}}{X / s-C}\right\}
$$

Substituting (2.8) into (2.5), we get

$$
\begin{gathered}
U=X f^{\prime}(Y)-\delta^{*} X^{2} f^{\prime \prime}(Y), \\
V=-f(Y)+2 \delta^{*} X f^{\prime}(Y) .
\end{gathered}
$$

Having obtained the analytical expression for the stream function $\psi$ and the velocity components $U$ and $V$, we now move on to discuss the results obtained in the study.

\section{Results and Discussion}

The problem of a flexible sheet undergoing quadratic stretching is investigated for the flow it generates in its immediate neighbourhood. The stretching sheet is the sole reason for the liquid flow, and liquid viscoelasticity significantly influences the flow. The flow is studied with the help of streamline patterns and also the axial and transverse velocity distributions. The results are analyzed against the background of the classical linear stretching problem $\left(\delta^{*}=0\right)$ involving Newtonian liquids $\left(k_{1}=0\right)$. Before we discuss the results of the study, we make some general observations. From (2.15) it is clear that the $k_{1}$ range of applicability of the solution is $(-\infty, 1)$. This can further be substantiated as follows. Differentiating equation (2.9) with respect to $Y$, and subject to condition (2.12a)-(2.12c), one gets

$$
f^{\prime \prime}(0)=\left(1-k_{1}\right) f^{\prime \prime \prime \prime}(0)
$$

From the above equation, we see that $f^{\prime \prime}(0)=0$ for $k_{1}=1$. In conjunction with the condition $f^{\prime \prime}(0)=-s$ in $(2.12 \mathrm{a})$, this would mean

$$
f^{\prime \prime}(0)=-s=0 .
$$




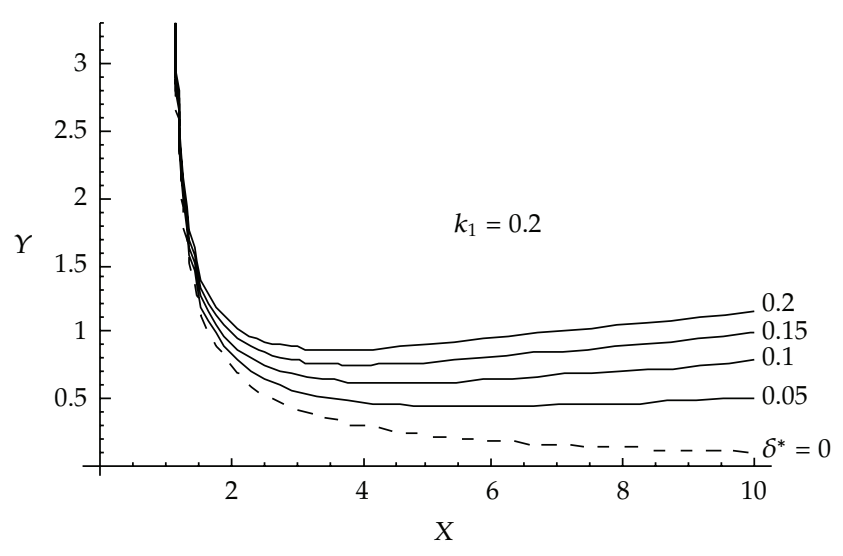

$\delta^{*}$ : Quadratic stretching parameter

$k_{1}$ : Viscoelastic parameter

Figure 2: Streamline $\psi(X, Y)=1$ for different values of quadratic stretching parameter $\delta^{*}$.

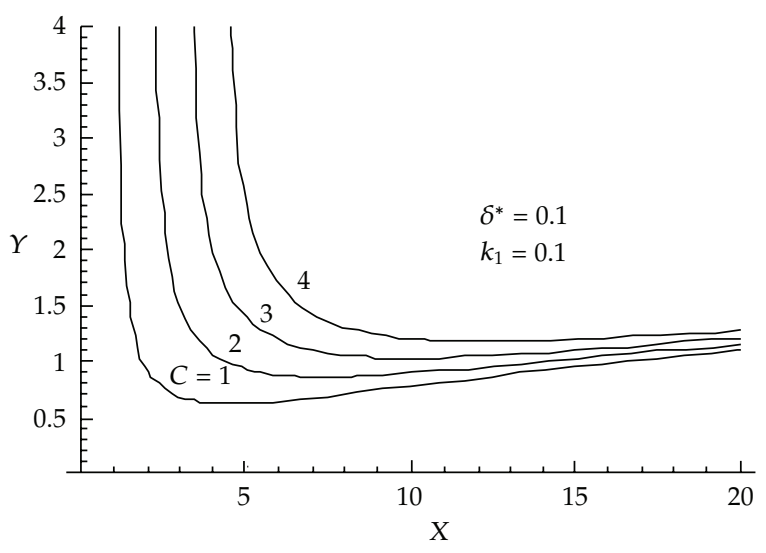

Figure 3: Streamlines $\psi(X, Y)=C$ for different values of $C$.

Obviously for $k_{1}=1$, the quadratic stretching problem ceases to exist. For $k_{1}>1$ we note that $s=1 / \sqrt{\left(1-k_{1}\right)}$ is complex. Hence it stands reiterated that the range of applicability of $k_{1}$ must be $(-\infty, 1)$.

We note that negative values of $k_{1}$ give us the results of a second order liquid and positive values of $k_{1}$ those of a Walters' liquid B model. We now discuss the results of the study on Walters' liquid B followed by those on the second order liquid.

Figure 2 is a plot of the streamline $\psi(X, Y)=1$ for different values of $\delta^{*}$ and $k_{1}=0.2$. Increasing value of $\delta^{*}$ indicates the increasing rate of quadratic stretching. We find from the figure that increasing rate of stretching restricts the dynamics in the axial direction to regions close to the slit.

Figure 3 is a plot of various stream lines $\psi(X, Y)=C$ when $\delta^{*}=0.1$ and $k_{1}=0.2$. As is depicted in the figure, at large axial distances the streamlines converge together and are lifted up due to quadratic stretching. 


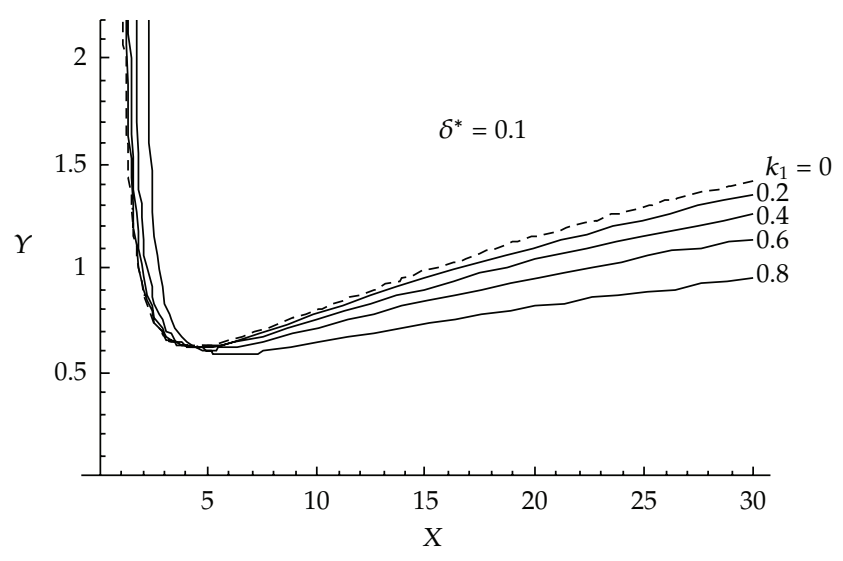

Figure 4: Streamline $\psi^{*}(X, Y)=1$ for different values of $k_{1}$.

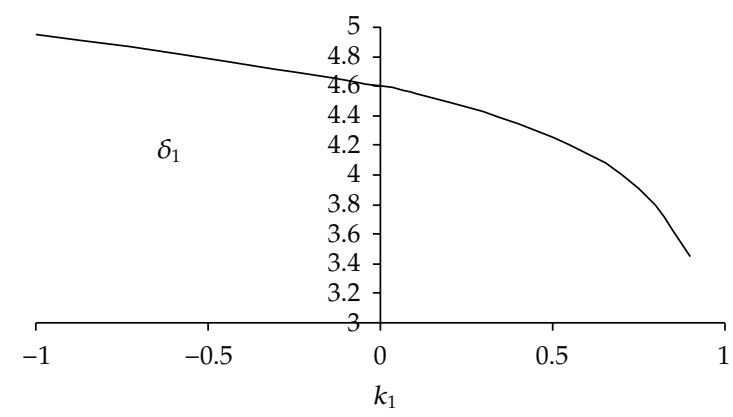

Figure 5: Variation of boundary layer thickness $\delta_{1}$ with $k_{1}$.

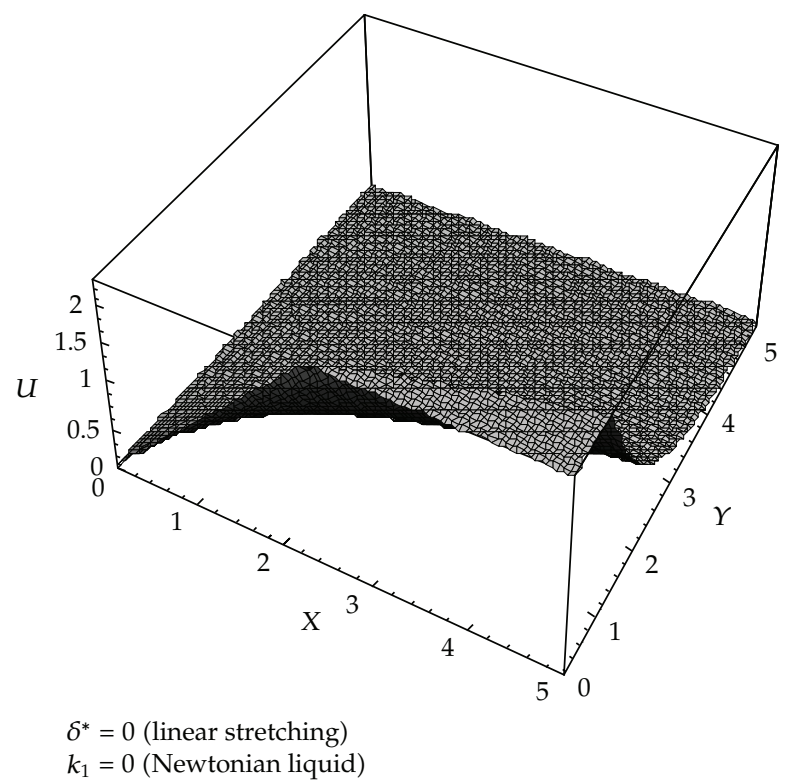

Figure 6: Axial velocity $U(X, Y)$ for the linear stretching sheet problem of Crane [19]. 


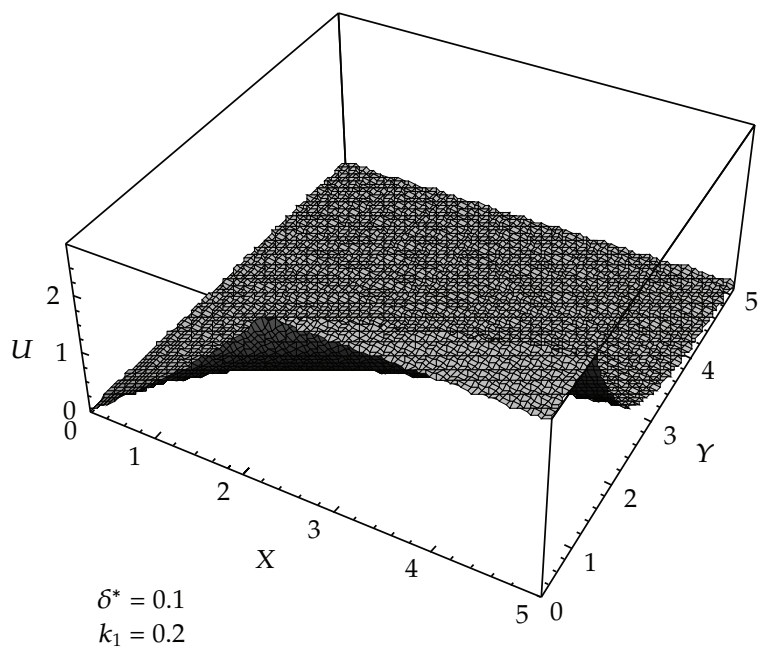

Figure 7: Axial velocity $U(X, Y)$ for the quadratic stretching sheet problem.

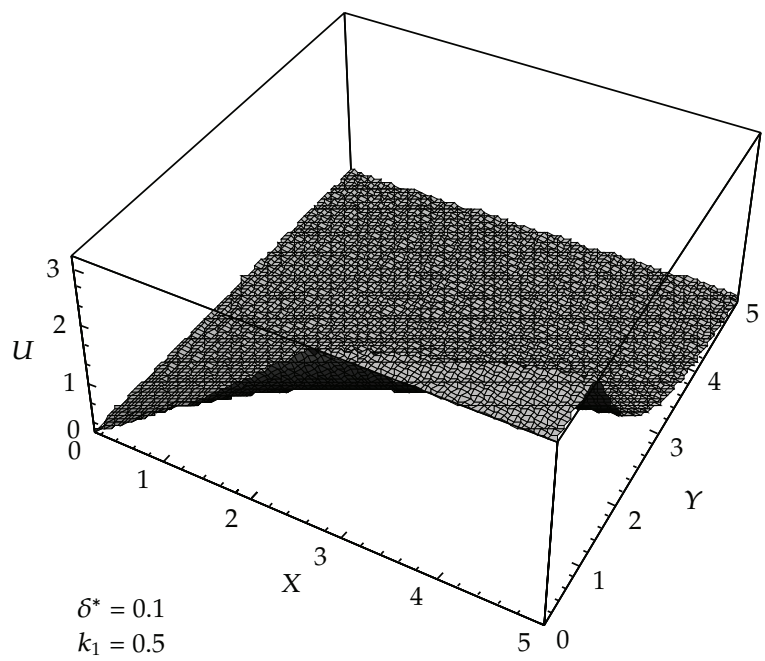

Figure 8: Axial velocity $U(X, Y)$ for the quadratic stretching sheet problem.

Figure 4 is a plot of the streamline $\psi(X, Y)=1$ for different values of $k_{1}$ and $\delta^{*}=$ 0.1. It is evident from the aforementioned 3 figures that the viscoelastic parameter $k_{1}$ and the quadratic stretching parameter $\delta^{*}$ work against each other in the lifting of the liquid as we go downstream. We now discuss the axial and transverse velocity distributions with an observation that

$$
\begin{gathered}
U=X e^{-s Y}+\delta^{*} s X^{2} e^{-s Y} \longrightarrow 0 \quad \text { as } Y \longrightarrow \infty, \\
V=-\frac{1-e^{-s Y}}{s}+2 \delta^{*} X e^{-s Y} \longrightarrow-\frac{1}{s} \text { as } Y \longrightarrow \infty,
\end{gathered}
$$

that is, the flow outside the boundary layer becomes uniform and is directed perpendicular to the sheet. The boundary layer thickness $\delta_{1}$, defined as the distance from the sheet at which 


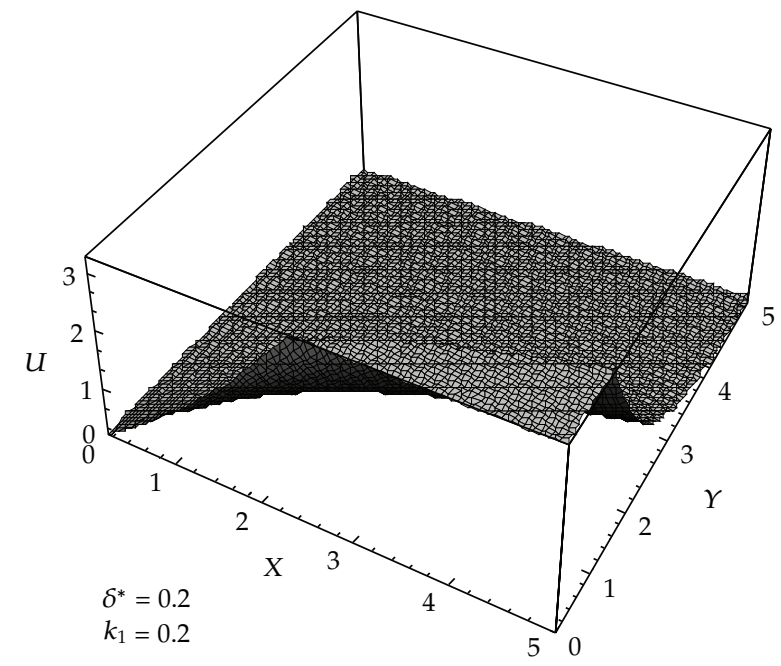

Figure 9: Axial velocity $U(X, Y)$ for the quadratic stretching sheet problem.

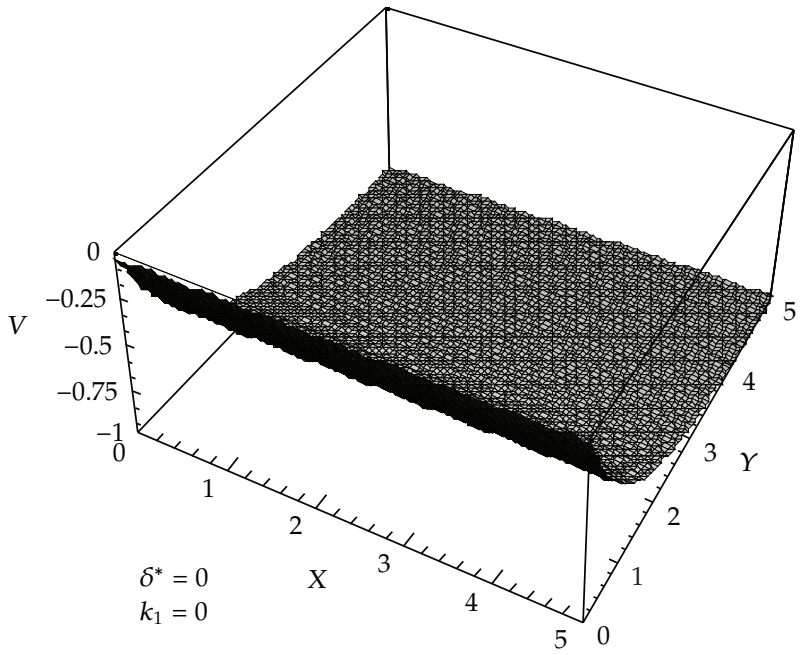

Figure 10: Transverse velocity $V(X, Y)$ for the linear stretching sheet problem of Crane [19].

the streamwise velocity $U$ has been reduced to one percent of the velocity $X+\beta^{*} X^{2}$ of the quadratic stretching surface, can be expressed as

$$
\delta_{1}=\operatorname{Ln}\left(\frac{100}{S}\right)=\operatorname{Ln}\left(100 \frac{\delta^{*}}{\beta^{*}}\right)
$$

Using (2.15) in the above equation, we get

$$
\delta_{1}=\operatorname{Ln}\left(100 \sqrt{1-k_{1}}\right)
$$




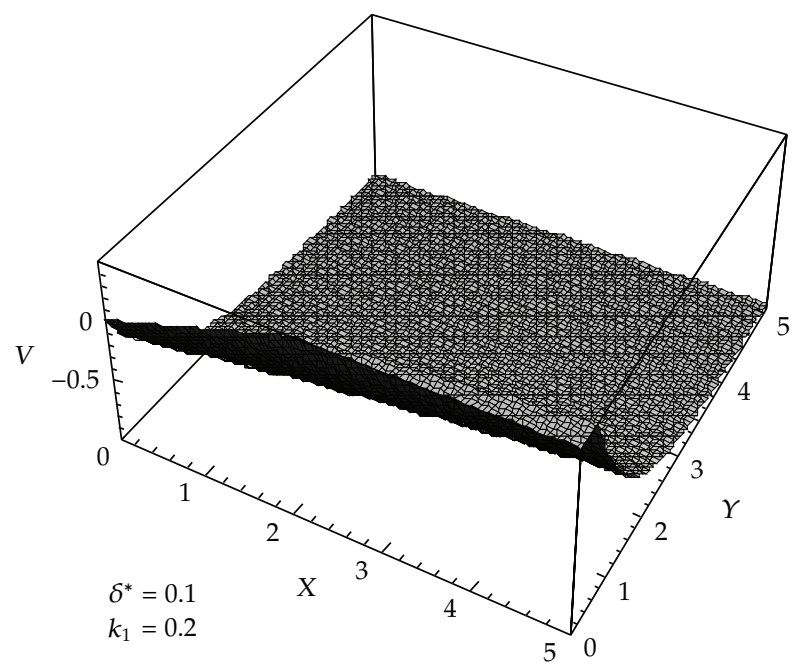

Figure 11: Transverse velocity $V(X, Y)$ for the quadratic stretching sheet problem.

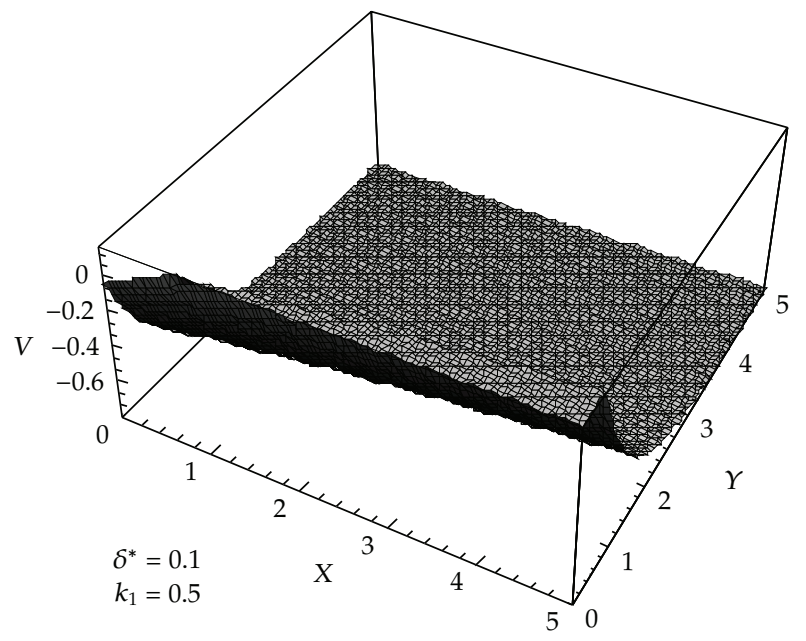

Figure 12: Transverse velocity $V(X, Y)$ for the quadratic stretching sheet problem.

Thus, we see that the boundary layer thickness $\delta_{1}$ is coordinate-independent for all permissible values of $k_{1}$. Figure 5 shows the variation of $\delta_{1}$ with $k_{1}$. We see from the figure that the effect of increasing $k_{1}$ is to decrease $\delta_{1}$.

Figures 6-15 that are three-dimensional plots of the velocity components $U(X, Y)$ and $V(X, Y)$ reveal more than the conventional two-dimensional projections on the $U-Y$ and $V-Y$ planes. Figure 6 is a plot of the Crane [19] profile of the linear stretching problem. One can easily see from the figure that the horizontal and vertical extent of the dynamics on the stretching sheet increases as we go along axial direction.

Figure 7 brings out the effect of the quadratic stretching of the sheet as well as the viscoelasticity of the liquid. Clearly both the above effects give rise to an extended dynamic region compared to the linear stretching problem of a Newtonian liquid. Comparing 


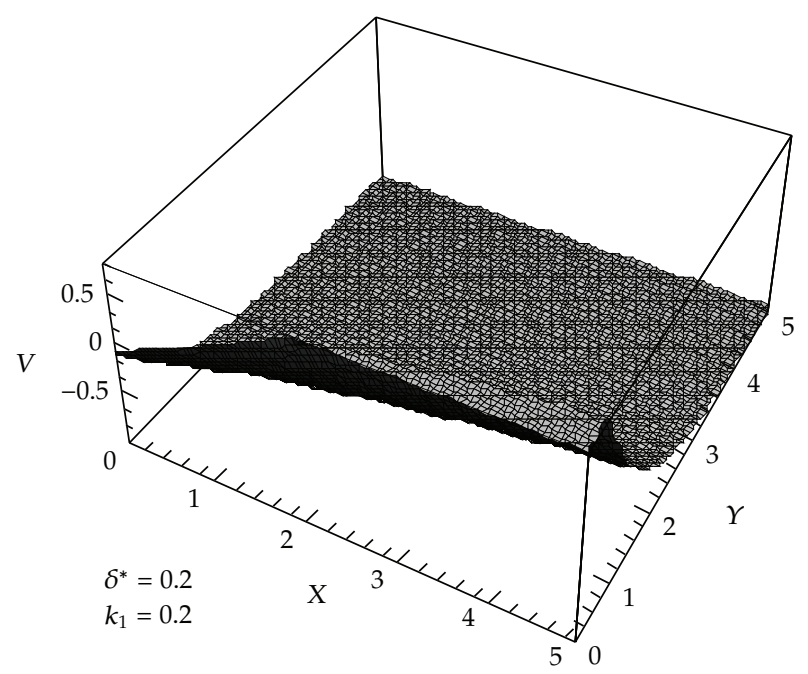

Figure 13: Transverse velocity $V(X, Y)$ for the quadratic stretching sheet problem.

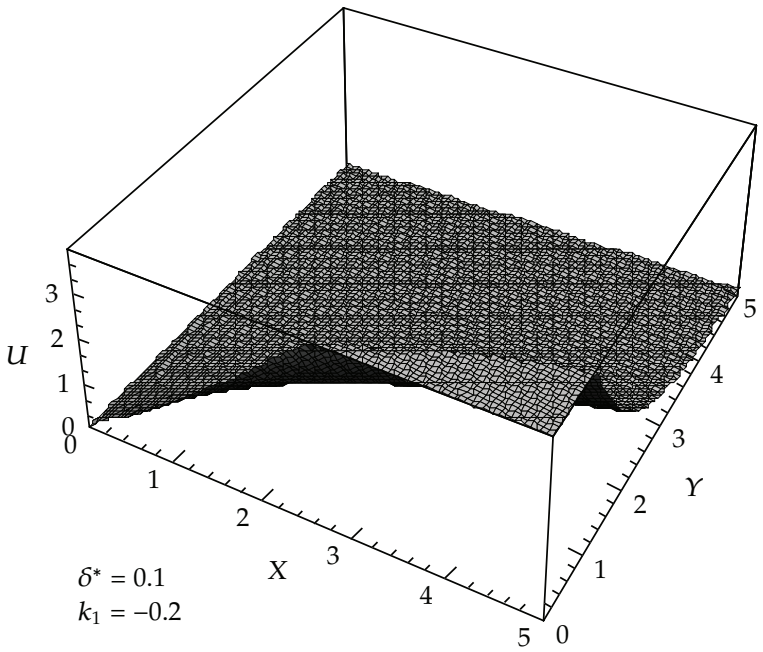

Figure 14: Axial velocity $U(X, Y)$ for the quadratic stretching sheet problem.

Figures 7 and 8 it is obvious that the quadratic stretching increases the vertical extent of the dynamic region. Comparing Figures 8 and 9 of quadratic stretching we find that the effect of viscoelasticity is to initiate lifting of the liquid more closer to the slit compared to that of a Newtonian liquid.

The transverse velocity profile brings out the fact that quadratic stretching greatly influences the vertical velocity compared to that in the case of linear stretching. Figure 10 depicts the $X$-independence of $V$ while Figure 11 spells out that quadratic stretching induces the $X$-dependence of the transverse velocity component $V$. Figures 12 and 13 explain the nature of the influence of $k_{1}$ on $V$ for the problem of quadratic stretching. The influence of $k_{1}$ on $V(X, Y)$ is similar to its influence on $U(X, Y)$ and the same is demonstrated by 


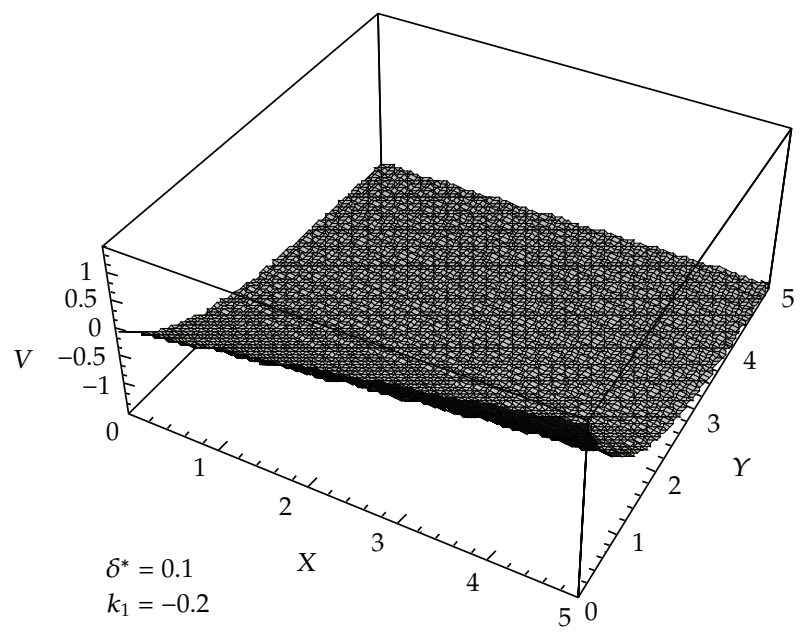

Figure 15: Transverse velocity $V(X, Y)$ for the quadratic stretching sheet problem.

Figures 12 and 13. The influence of quadratic stretching on $U(X, Y)$ and $V(X, Y)$ of a secondorder liquid shows that the vertical variation is comparatively less than axial variation.

Figures 14 and 15 are the axial and transverse velocity profiles for the quadratic stretching sheet problem in a second-order liquid. The corresponding graphs for a Walters' liquid B model are Figures 7 and 11. It is clear that the lifting is initiated closer to the slit in the case of a Walters' liquid B model compared to the second-order liquid. In the case of the latter the flow is more strongly two dimensional than in the former case. This is clearly seen on comparing Figures 11 and 15.

\section{References}

[1] B. C. Sakiadis, "Boundary-layer behavior on continuous solid surfaces, I. The boundary layer equations for two dimensional and axi-symmetric flow," AIChE Journal, vol. 7, pp. 26-28, 1961.

[2] B. C. Sakiadis, "Boundary-layer behavior on continuous solid surfaces, II. The boundary layer on a continuous flat surface," AIChE Journal, vol. 7, pp. 221-225, 1961.

[3] P. G. Siddheshwar and U. S. Mahabaleswar, "Effects of radiation and heat source on MHD flow of a viscoelastic liquid and heat transfer over a stretching sheet," International Journal of Non-Linear Mechanics, vol. 40, no. 6, pp. 807-820, 2005.

[4] P. G. Siddheshwar and U. S. Mahabaleswar, "A new analytical procedure for solving the nonlinear differential equation arising in the stretching sheet problem," Journal of Differential Equations. resubmitted.

[5] H. I. Andersson, "MHD flow of a viscoelastic fluid past a stretching surface," Acta Mechanica, vol. 95, no. 1-4, pp. 227-230, 1992.

[6] H. I. Andersson, K. H. Bech, and B. S. Dandapat, "Magnetohydrodynamic flow of a power-law fluid over a stretching sheet," International Journal of Non-Linear Mechanics, vol. 27, no. 6, pp. 929-936, 1992.

[7] H. I. Andersson, "An exact solution of the Navier-Stokes equations for magnetohydrodynamic flow," Acta Mechanica, vol. 113, no. 1-4, pp. 241-244, 1995.

[8] D. Rollins and K. Vajravelu, "Heat transfer in a second-order fluid over a continuous stretching surface," Acta Mechanica, vol. 89, no. 1-4, pp. 167-178, 1991.

[9] J. Vleggaar, "Laminar boundary-layer behaviour on continuous, accelerating surfaces," Chemical Engineering Science, vol. 32, no. 12, pp. 1517-1525, 1977. 
[10] C. Ming-I and C. Cha'o-Kuang, "Temperature field in non-Newtonian flow over a stretching plate with variable heat flux," International Journal of Heat and Mass Transfer, vol. 31, no. 5, pp. 917-921, 1988.

[11] D. Kelly, K. Vajravelu, and L. Andrews, "Analysis of heat and mass transfer of a viscoelastic, electrically conducting fluid past a continuous stretching sheet," Nonlinear Analysis: Theory, Methods E Applications, vol. 36, no. 6, pp. 767-784, 1999.

[12] K. Vajravelu and A. Hadjinicolaou, "Heat transfer in a viscous fluid over a stretching sheet with viscous dissipation and internal heat generation," International Communications in Heat and Mass Transfer, vol. 20, no. 3, pp. 417-430, 1993.

[13] S. J. Liao and I. Pop, "Explicit analytic solution for similarity boundary layer equations," International Journal of Heat and Mass Transfer, vol. 47, no. 1, pp. 75-85, 2004.

[14] E. Magyari, I. Pop, and B. Keller, “New analytical solutions of a well-known boundary value problem in fluid mechanics," Fluid Dynamics Research, vol. 33, no. 4, pp. 313-317, 2003.

[15] S.-J. Liao, "On the analytic solution of magnetohydrodynamic flows of non-Newtonian fluids over a stretching sheet," Journal of Fluid Mechanics, vol. 488, pp. 189-212, 2003.

[16] B. S. Dandapat and A. S. Gupta, "Flow and heat transfer in a viscoelastic fluid over a stretching sheet," International Journal of Non-Linear Mechanics, vol. 24, no. 3, pp. 215-219, 1989.

[17] K. R. Rajagopal, T. Y. Na, and A. S. Gupta, "Flow of a viscoelastic fluid over a stretching sheet," Rheologica Acta, vol. 23, no. 2, pp. 213-215, 1984.

[18] D. W. Beard and K. Walters, "Elastico-viscous boundary-layer flows. I. Two-dimensional flow near a stagnation point," Proceedings of the Cambridge Philosophical Society, vol. 60, pp. 667-674, 1964.

[19] L. J. Crane, "Flow past a stretching plate," Zeitschrift für angewandte Mathematik und Physik ZAMP, vol. 21, no. 4, pp. 645-647, 1970. 


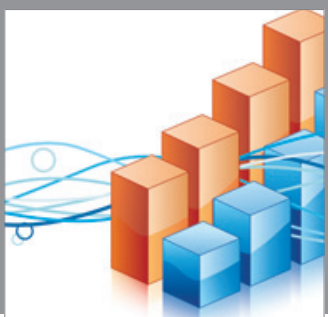

Advances in

Operations Research

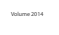

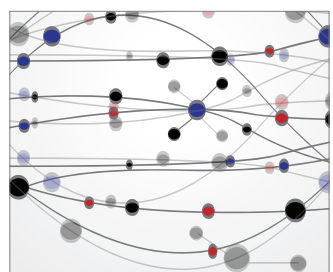

\section{The Scientific} World Journal
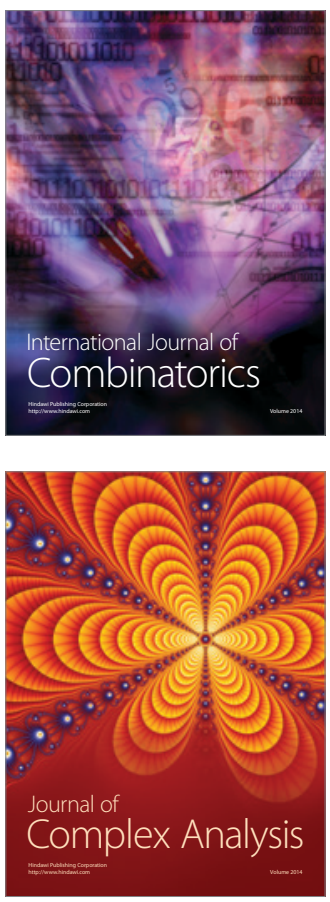

International Journal of

Mathematics and

Mathematical

Sciences
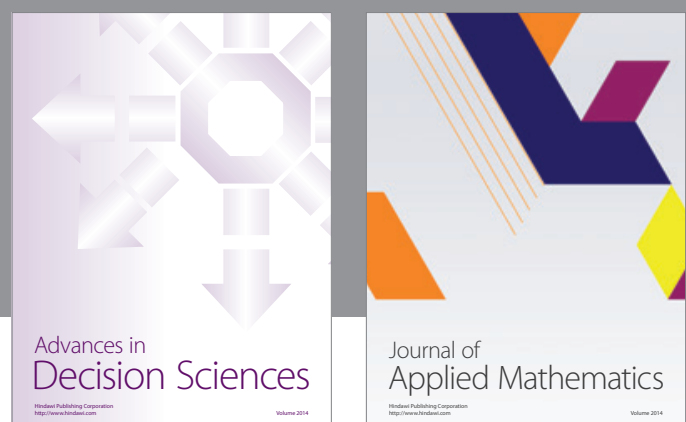

Journal of

Applied Mathematics
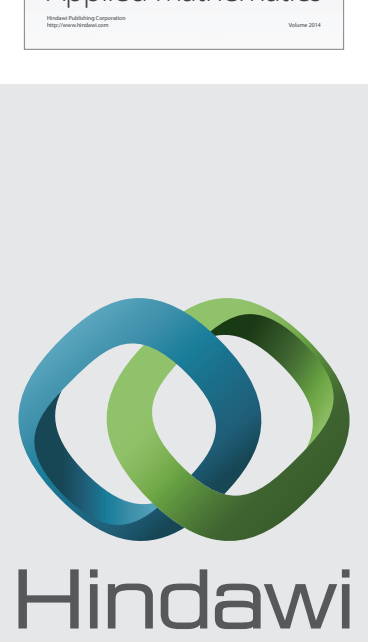

Submit your manuscripts at http://www.hindawi.com
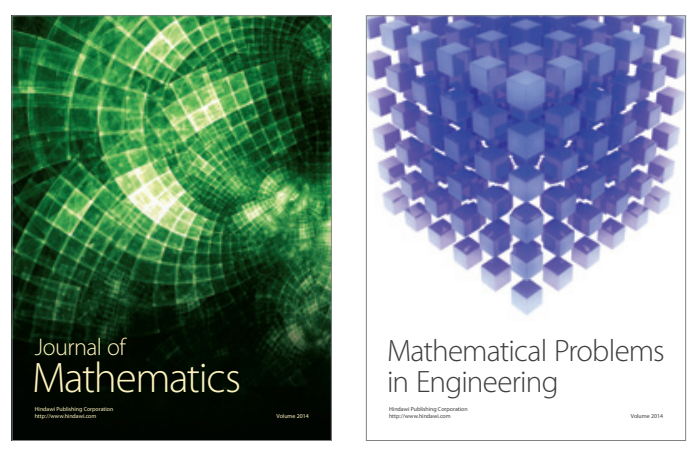

Mathematical Problems in Engineering
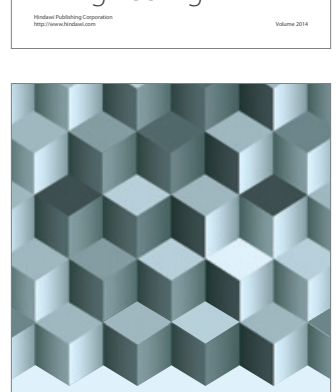

Journal of

Function Spaces
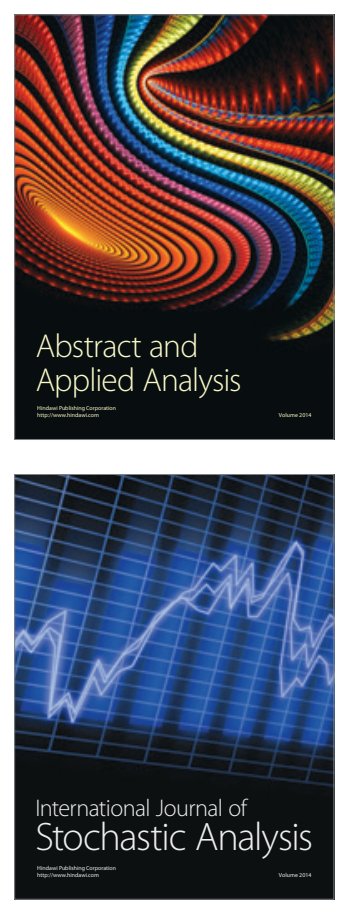

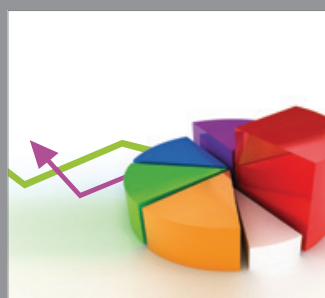

ournal of

Probability and Statistics

Promensencen
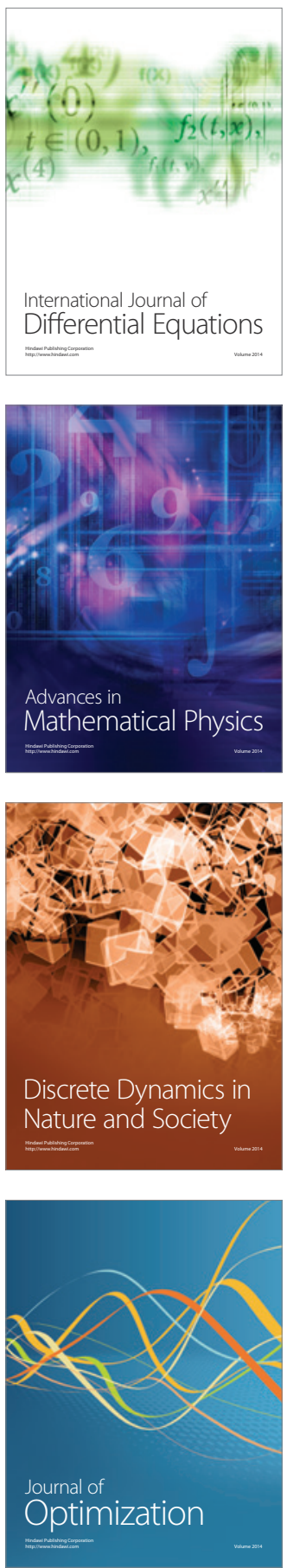